\title{
Artificial Infection of Rosellinia necatrix with Purified Viral Particles of a Member of the Genus Mycoreovirus Reveals Its Uneven Distribution in Single Colonies
}

\author{
Atsuko Sasaki, Satoko Kanematsu, Mari Onoue, Yuri Oikawa, Hitoshi Nakamura, and Kouji Yoshida
}

First, fifth, and sixth authors: Department of Plant Protection, National Institute of Fruit Tree Science, National Agricultural Research Organization (NARO), Independent Administrative Institution, 2 Fujimoto, Tsukuba, Ibaraki 305-8605, Japan; and second, third, and fourth authors: Department of Apple Research, National Institute of Fruit Tree Science, National Agricultural Research Organization (NARO), Independent Administrative Institution, 92 Shimokuriyagawa, Morioka, Iwate 020-0123, Japan.

Accepted for publication 23 August 2006.

\begin{abstract}
Sasaki, A., Kanematsu, S., Onoue, M., Oikawa, Y., Nakamura, H., and Yoshida, K. 2007. Artificial infection of Rosellinia necatrix with purified viral particles of a member of the genus Mycoreovirus reveals its uneven distribution in single colonies. Phytopathology 97:278-286.

Rosellinia necatrix mycoreovirus 3 (W370) (RnMYRV-3/W370, described as RnMYRV-3 in this paper), a member of the newly established genus Mycoreovirus within the family Reoviridae, is the hypovirulence factor of the white root rot fungus, Rosellinia necatrix. Two virus-free fungal isolates (W37 and W97) that were somatically incompatible with the virus-harboring field isolate (W370) were transfected with purified RnMYRV-3 particles. Virus infection was confirmed by electrophoresis and northern hybridization of viral double-stranded RNA. RnMYRV-3

was transmissible from transfected strains to their respective, virus-free counterparts via hyphal anastomosis. Virus-transfected strains produced smaller lesions on apple fruits than did their virus-free counterparts. Virus-cured strains were indistinguishable from wild-type strains in culture morphology and displayed approximately the same virulence level on apples. Virus-transfected strains had "mosaic" colony portions consisting of thin, fast-growing and dense, slow-growing mycelia, and grew more slowly as a whole than their virus-free, parental strains. The level of virus accumulation varied among virus-transfected subcultures and within its single colonies. Virus-transfected strains were occasionally cured, as was W370. Such a phenomenon may be ascribed to uneven viral distribution in single colonies and the difficulty in viral transmission to virus-free hyphae.
\end{abstract}

Mycoviruses are widespread throughout the major taxonomic groups of fungi (5), and most mycoviruses with double-stranded (ds)RNA genome are classified into five families, Totiviridae, Partitiviridae, Chrysoviridae, Hypoviridae, and Reoviridae, according to their genomic constitution and viral particle properties $(9,10,23,25,38)$. In many cases, they exhibit no apparent symptoms on host fungi (5), but several viral species are known to reduce the virulence of phytopathogenic fungi, a phenomenon referred to as hypovirulence $(1,4,6,13,16,18,20,21,27)$. Those estimations of hypovirulence sometimes hampered because mycoviruses are transmitted exclusively through hyphal anastomosis and artificial infection is regarded as generally intractable (5). The practical application of hypovirulence is difficult because viral horizontal transmission is prevented by somatic incompatibility within the same species (1), and viruses are sometimes lost during sexual and/or asexual reproduction $(1,6)$.

Rosellinia necatrix Prill. is the causal agent of white root rot of 171 plant species belonging to 63 genera $(33,34)$, including many kinds of fruit trees. The disease may be controlled by drenching 50 to 200 liters of fungicide per tree every other year (19), causing concerns about soil pollution. Consequently, an attempt to control $R$. necatrix with mycoviruses was proposed because of the simple population structure of $R$. necatrix, which allows efficient spread of biocontrol agents (22). In addition, the spread of the fungus in orchards mainly depends on mycelial growth, which would overcome the curing mechanisms by sporulation

Corresponding author: A. Sasaki; E-mail address: sasaatu@affrc.go.jp

DOI: 10.1094/PHYTO-97-3-0278

(C) 2007 The American Phytopathological Society
(24). More than 400 isolates of $R$. necatrix have been collected throughout Japan, and 82 were found to harbor dsRNAs varying in size and number of segments $(2,17)$. Of the 82 isolates, W8 and W370 were studied in particular because of their weak virulence.

The family Reoviridae consists of 12 genera, and accommodates members that infect a broad range of host species, such as vertebrates, arthropods, plants, and fungi; therefore, it is regarded as one of the largest family in virus taxon (23). The genus Mycoreovirus has recently been included within the family Reoviridae. One and two reoviruses were reported from phytopathogenic fungi, $R$. necatrix $(26,36,37)$ and Cryphonectria parasitica $(7,11$, 32 ), respectively. The former is termed Rosellinia necatrix mycoreovirus 3 (W370) (RnMYRV-3/W370) and the latter Cryphonectria parasitica mycoreovirus 1 (9B21) (CpMYRV-1/ 9B21) and Cryphonectria parasitica mycoreovirus 2 (C18) (CpMYRV-2/C18) in the 8th Report of the International Committee on Taxonomy of Viruses (and hereafter referred to RnMYRV-3, CpMYRV-1, and CpMYRV-2, respectively). RnMYRV-3 and CpMYRV-1 were studied in detail. Both mycoreoviruses have typical features of the family Reoviridae, and encode similar proteins that show the identity of amino acid sequence ranging from 14.7 to $34.2 \%$. Importantly, both mycoviruses were reported as hypovirulence factors $(11,20)$, but their biological behavior in their respective host fungus is different. Although CpMYRV-1 was stable in mycelia and readily transmissible by hyphal anastomosis, isolate W370, naturally infected with RnMYRV-3, sometimes lost the virus during subculture and inoculation experiments of plants. Its transmission from W370 to the opposite, virus-free counterpart in dual culture was limited even though they were somatically compatible (20). The unstable nature of RnMYRV-3 infection may be similar to that reported for 
CpMYRV-2, although RNA-dependent RNA polymerase of CpMYRV-2 was more closely related to CpMYRV-1 than to RnMYRV-3 (12).

We established a transfection protocol to study cause-effect relationships in the RnMYRV-3/R. necatrix system and unstable infection in the host fungus. Purified particles of RnMYRV-3 were infectious to virus-free $R$. necatrix as with CpMYRV-1 and CpMYRV-2 to $C$. parasitica (11). Transfected isolates of $R$. necatrix indicated reduced virulence, but RnMYRV-3 remained unstable. We further showed that viral distribution in single colonies was irregular and that the phenomenon is considered to be related to spontaneous cure from viral infection during subculture.

\section{MATERIALS AND METHODS}

Fungal isolates and culture condition. $R$. necatrix isolates W370 (containing RnMYRV-3), W37, and W97 (both virus-free), described in a previous paper, were used (2). Isolates W37 and W97 were somatically incompatible with each other and with W370. A virus-cured strain derived from W370 (W370T1) was obtained by Kanematsu et al. (20). Isolates W37, W97, and W370T1 were transformed with the bacterial hygromycin resistance gene $(h p h)$ (each designated as W37hyg, W97hyg, and RT37-1, respectively) for viral transmission assay $(20,28)$. Viruscured strains from transfected W37 and W97 were obtained by hyphal tipping. All R. necatrix strains were cultured on potato dextrose agar (PDA) (Difco Laboratories, Detroit) with or without hygromycin at $20^{\circ} \mathrm{C}$ in the dark and stored at $4{ }^{\circ} \mathrm{C}$ until use. Virustransfected strains were maintained on cellophane-overlaid PDA plates to confirm the presence of viral dsRNA in mycelia before subculture. All transfected strains were subcultured about every 10 days to keep virus infection. Four small mycelial plugs were obtained from each side of the rectangular PDA plate covered with cellophane and subcultured. Colonies were cultured in $12 \mathrm{~cm}$ square dishes containing $30 \mathrm{ml}$ of PDA entirely overlaid with $11.5 \mathrm{~cm}$ square of cellophane to determine the presence of the virus.

Transfection of fungal protoplast with purified viral particles. RnMYRV-3 particles were prepared as described previously (36) with a slight modification, excluding Triton X-100 treatment. The purified viral particle fraction from approximately 20 to $24 \mathrm{~g}$ (wet weight) of fungal mycelia was suspended in $20 \mu \mathrm{l}$ of $0.05 \mathrm{M}$ phosphate buffer ( $\mathrm{pH}$ 7.0), filtrated through Ultrafree-MC sterile centrifugal filter units (Millipore, Tokyo, Japan), and subjected to infection assay. The filtrated fraction was introduced to a suspension containing 1.7 to $3.8 \times 10^{6}$ of protoplasts with polyethylene glycol according to the methods described by Sasaki et al. (28). Viral dsRNA in regenerated colonies was detected as previously described (28).

Extraction of nucleic acids. Mycelia harvested from cellophane-overlaid PDA were used to extract nucleic acids, according to Sasaki et al. (29). Total DNA was prepared from total nucleic acids by overnight treatment with $10 \mu \mathrm{g}$ of RNase A in Tris-EDTA at $4{ }^{\circ} \mathrm{C}$. The reaction was stopped by incubating at $55^{\circ} \mathrm{C}$ for 10 min with additional sodium dodecyl sulfate at $2 \%$ final concentration. Total DNA was precipitated with $0.2 \mathrm{M} \mathrm{NaCl}$ and 2 volumes of $\mathrm{EtOH}$ at room temperature, and immediately recovered by centrifugation at $5,000 \times g$ for $5 \mathrm{~min}$ at $20^{\circ} \mathrm{C}$. The concentration of total DNA was calculated by Gene Spec V spectrophotometer (Hitachi, Tsukuba, Japan). Total nucleic acids were treated with both DNase and S1 nuclease as described by Sasaki et al. (29) or singly with 20 units of S1 nucleae to visualize dsRNA by electrophoresis.

Electrophoretic analysis. Extracted dsRNA was electrophoresed in $5 \%$ polyacrylamide gel electrophoresis gel as described by Osaki et al. (26) to confirm the existence of all segments in transfected strains, or in Tris-acetate-EDTA (TAE)$0.7 \%$ agarose to check for the presence of RnMYRV-3 in fungal colonies. To determine viral distribution, $2 \mu \mathrm{g}$ of total DNA and dsRNA contained in $4 \mu \mathrm{g}$ of total DNA fraction was electrophoresed in TAE- $0.7 \%$ agarose. The gels were stained with ethidium bromide for visual observation after electrophoresis.

Northern analysis. Extracted dsRNA was electrophoresed in TAE- $0.7 \%$ agarose, denatured in gel, and blotted as described by Sasaki et al. (29). Northern analysis was performed according to Ikeda et al. (15) using direct labeling probes prepared from RnMYRV-3.

Viral transmission in paired culture. A virus-transfected mycelial plug (donor) was inoculated onto a $12 \mathrm{~cm}^{2}$ square dish containing $30 \mathrm{ml}$ of PDA and incubated at $20^{\circ} \mathrm{C}$. After 2 or 3 days, a virus-free mycelial plug (recipient), which had been transformed with $h p h$ gene was inoculated near the donor colony and incubated further for 19 to 21 days. Mycelial plugs were removed from both colonies of recipient and donor and subcultured on PDA plates as described by Sasaki et al. (28).

Virulence estimation of transfected strains. Virulence was assayed on apple fruits (Malus pumila var. domestica cv. Fuji). A small area of apple skin (approximately 5-mm diameter) was peel with a cork borer, and a mycelial plug from the fungal colony was applied to the peeled area. Inoculated apples were covered with polyethylene bags and incubated at $20^{\circ} \mathrm{C}$ for 3 weeks in the dark. The lesion was measured as follows: lesion $=1 / 2$ lengthwise $\times$ $1 / 2$ crosswise $\times 3.14$.

\section{RESULTS}

Viral transfection to virus-free isolates. The dsRNA fraction extracted from wild-type isolates W37 and W97 did not indicate the presence of viral genomic segments (Fig. 1A).

Regenerated strains of W37 and W97 after transfection with purified RnMYRV-3 contained 12 segments of dsRNAs identical to those of W370. Two strains infected with RnMYRV-3 for isolates W37 and W97 were obtained by two independent transfection experiments. These dsRNA segments hybridized with direct-labeled RnMYRV-3 dsRNA extracted from W370 (Fig. 1B). Hybridization was not observed in the wild-type strains. Viral dsRNA disappeared in virus-cured strains as shown by both electrophoresis gel and northern hybridization (Fig. 1A and B). Transfection frequency ranged from 6.25 to $12.5 \%$ in both isolates (Table 1).

Effect of viral infection on fungal growth. We obtained two virus-transfected strains each of W37 and W97 by independent transfection experiments. Virus-cured strains of W37 and W97 indicated the same morphology and growth rate as that of wild types (Fig. 2A). Strains W37(RnMYRV-3) and W97(RnMYRV-3) had dense mycelia as observed in isolate W370 infected with RnMYRV-3, but colonies of transfected strains also infrequently contained dispersed, fast-growing mycelia. Virus-transfected strains obviously showed reduced growth compared with the wild types (Fig. 2A and B); however, the virus disappeared in transfected W37 and W97 occasionally, as in W370. Although the presence of viral dsRNA was confirmed in primary cultures, it sometimes disappeared in secondary cultures (Fig. 2C). For example, colony 2 of strain W37(RnMYRV-3)-I in the left panel of Figure 2B contained viral dsRNA (Fig. 2C), but its derivatives varied in the amount of dsRNA. The colony of secondary culture 4 of strain W37(RnMYRV-3)-I indicated reduced growth compared with the wild type, but dsRNA was not observed by electrophoresis under this condition (Fig. 2C, right panel). As shown in Figure 2B and C, subcultures of W37(RnMYRV-3) and W97(RnMYRV-3) varied in colony morphology and in the amount of dsRNA, but there was no clear relationship between them.

Viral distribution in single colonies. RnMYRV-3 sometimes disappeared even though its presence in the primary culture had been confirmed. We subsequently determined the viral distribution pattern in single colonies. W37(RnMYRV-3) and W97(RnMYRV- 
3) were cultured on PDA overlaid with cellophane in $12 \mathrm{~cm}$ square plates. Colonies were divided into 10 segments as shown in Figure 3, and total DNA and viral dsRNA were prepared from each. In Figure 4, $2 \mu \mathrm{g}$ of total DNA fraction from each colony segment indicated almost the same band intensity in agarose gel, but viral dsRNA fractions from those containing $4 \mu \mathrm{g}$ of total DNA fractions varied in band intensity. W37(RnMYRV-3)-I and W97(RnMYRV-3)-V exhibited relatively strong dsRNA signals from entire colony segments, but W37(RnMYRV-3)-II and W97(RnMYRV-3)-IV contained weak dsRNA signals. There was no obvious relationship between culture morphology and dsRNA distribution pattern within single colonies.

Viral transmission in dual culture. Viral transmission was determined using W37(RnMYRV-3)-I and W97(RnMYRV-3)-V as donors, and their respective, virus-free counterparts as recipients in paired culture (Fig. 5). Donors were inoculated 2 or
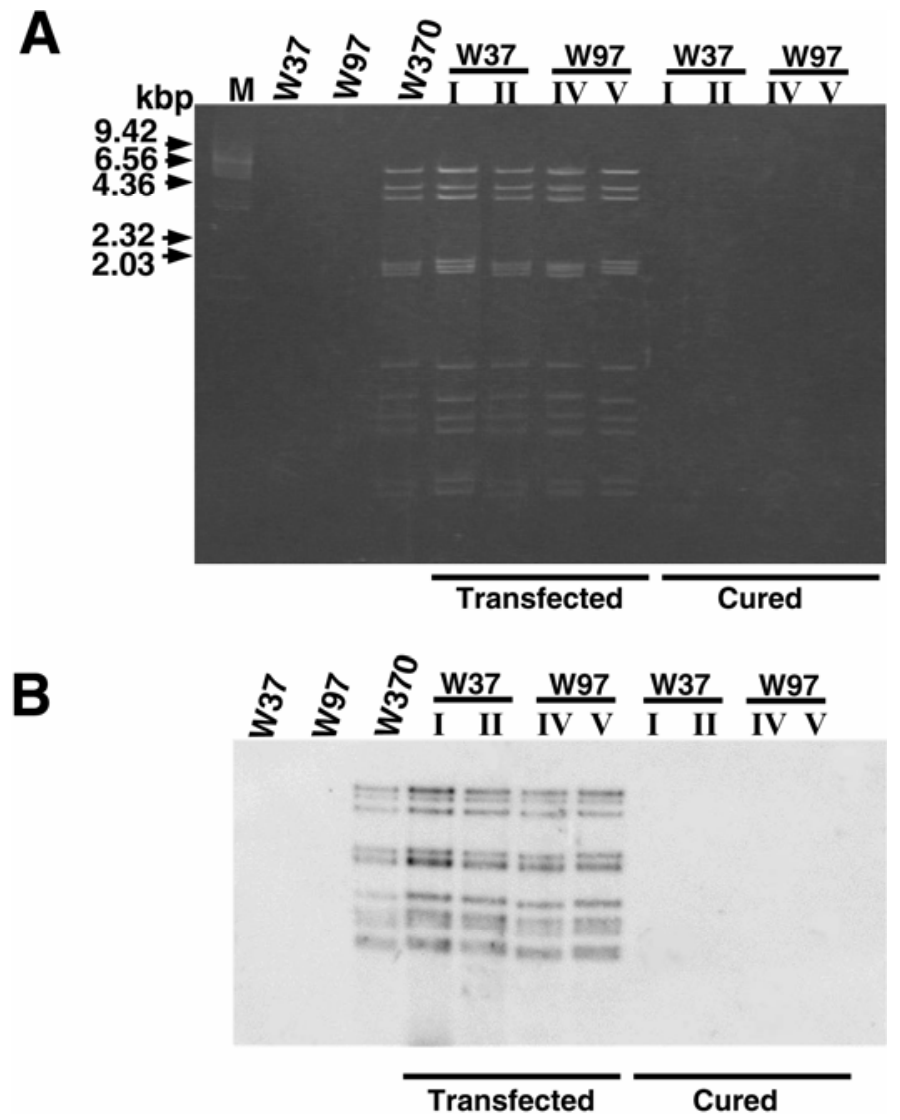

Fig. 1. Polyacrylamide gel electrophoresis and northern hybridization of double-stranded (ds)RNA in Rosellinia necatrix strains W37 and W97 transfected with Rosellinia necatrix mycoreovirus 3. Extracted dsRNA was electrophoresed on $\mathbf{A}, 5 \%$ polyacrylamide gel or $\mathbf{B}, 0.7 \%$ agarose in Tris-acetateEDTA. Isolates and strains are indicated above the lanes. Lane M is lambdaphage DNA digested with HindIII as a molecular marker. Treatment (virustransfected or -cured) is indicated under the lanes. Direct-labeled dsRNA probe used in northern analysis was prepared according to Ikeda et al. (15).

TABLE 1. Frequency of viral infection with purified Rosellinia necatrix mycoreovirus 3 (RnMYRV-3) particles in Rosellinia necatrix

\begin{tabular}{lcc}
\hline Isolate & Experiment $1^{\mathrm{a}}$ & Experiment $2^{\mathrm{a}}$ \\
\hline W37 & $1 / 16(6.25 \%)$ & $2 / 16(12.5 \%)$ \\
W97 & $1 / 16(6.25 \%)$ & $1 / 16(6.25 \%)$ \\
\hline
\end{tabular}

a The presence of RnMYRV-3 double-stranded RNA was examined on eight colonies each from two regeneration plates by agarose gel electrophoresis. Number of colonies containing RnMYRV-3/number of colonies examined. Number in parenthesis indicates percent infected.
3 days before recipients to prevent the overgrowth of recipients with fast mycelial growth. The growth of recipient colonies was slightly reduced compared with that in incompatible combinations, but morphological changes were not remarkable (Fig. 5A). Subcultures from recipients had a reduced growth rate and dense mycelia as donors when RnMYRV-3 was transmitted (Fig. 5B and C). On the other hand, when the combination was incompatible, the typical incompatible reaction (black line at colony junction) was observed, and finally, donors were surrounded by recipients (Fig. 5A, lower panel). The transmission of RnMYRV-3 from W370 to virus-free, incompatible recipients did not occur, and subcultures from recipient colonies did not show any appreciable change in colony morphology or dsRNA segments in agarose gel (Fig. 5B and C).

Effect of RnMYRV-3 on fungal virulence. The effect of RnMYRV-3 on fungal virulence was estimated based on the size of the lesion formed on apple fruits. Browned epidermis around the inoculation point was evident 3 days after inoculation, followed by black, round to elliptical rot. The lesion caused by W370 was smaller than the virus-cured strain (Fig. 6; Table 2). Relative lesion size to virus-free counterparts ranged between 38.3 and $47.0 \%$ in two separate experiments. Lesions caused by W37(RnMYRV-3) and W97(RnMYRV-3) were also smaller than by their wild types. The reduction ratio by virus infection ranged from 25.1 to $60.9 \%$ in $\mathrm{W} 37$ and from 35.2 to $80.0 \%$ in W97. W37(RnMYRV-3)-I and W97(RnMYRV-3)-V formed smaller lesions than did W37(RnMYRV-3)-II and W97(RnMYRV-3)-IV. Virus-cured strains caused lesions of almost the same size to those of their wild-type counterparts. When virus-free strains were inoculated, white mycelia typical to $R$. necatrix were sometimes observed on the surface of lesions and epidermis but were rare in the case of virus-transfected strains. Re-isolation of virustransfected strains from small lesions revealed the presence of viral dsRNA, but large lesions equivalent to those by virus-free strains did not reveal dsRNA (data not shown).

\section{DISCUSSION}

Transfection of fungal hosts by particles of a mycovirus is generally hampered by the difficulty in virus particle purification and/or unavailability of a reliable method for their introduction to host cells. There are a limited number of reports in which mycoviruses are shown to be infectious as virus particles. Examples include CpMYRV-1 and CpMYRV-2, infecting C. parasitica $(11,12)$, and a partitivirus, RnPV-1/W8 infecting $R$. necatrix (28). We succeeded in transfecting the hypovirulent factor, RnMYRV-3, belonging to the family Reoviridae. Electrophoresis and northern hybridization demonstrated that RnMYRV-3 infected two virus-free isolates of $R$. necatrix. The success of infection was also supported by viral transmission assay. Thus, this is an additional example of mycovirus transfection into a filamentous fungus; however, a difference was found between the results in these and previous transfection studies. In contrast to the high efficiency for transfection for CpMYRV-1 or RnPV-1/W8, the infection frequency of RnMYRV3 was low. In the case of RnPV-1/W8 transfection, the same isolates W37 and W97 as in this study become infected at approximately $100 \%$ (28). The difference may be ascribed to the following reasons. (i) Protoplasts transfected with the virus are considered to seldom regenerate. (ii) Kanematsu et al. (20) described that RnMYRV-3 transmission was limited to the area adjacent to the donor in paired culture. The difficulty of viral transmission suggested that the virus failed to spread through whole regenerated mycelia from infected protoplasts. (iii) The viability of the virus may be influenced by the purification procedure. We followed the methods of Wei et al. (36) to obtain purified viral fraction, but this method may not retain enough infectivity of the virus. 


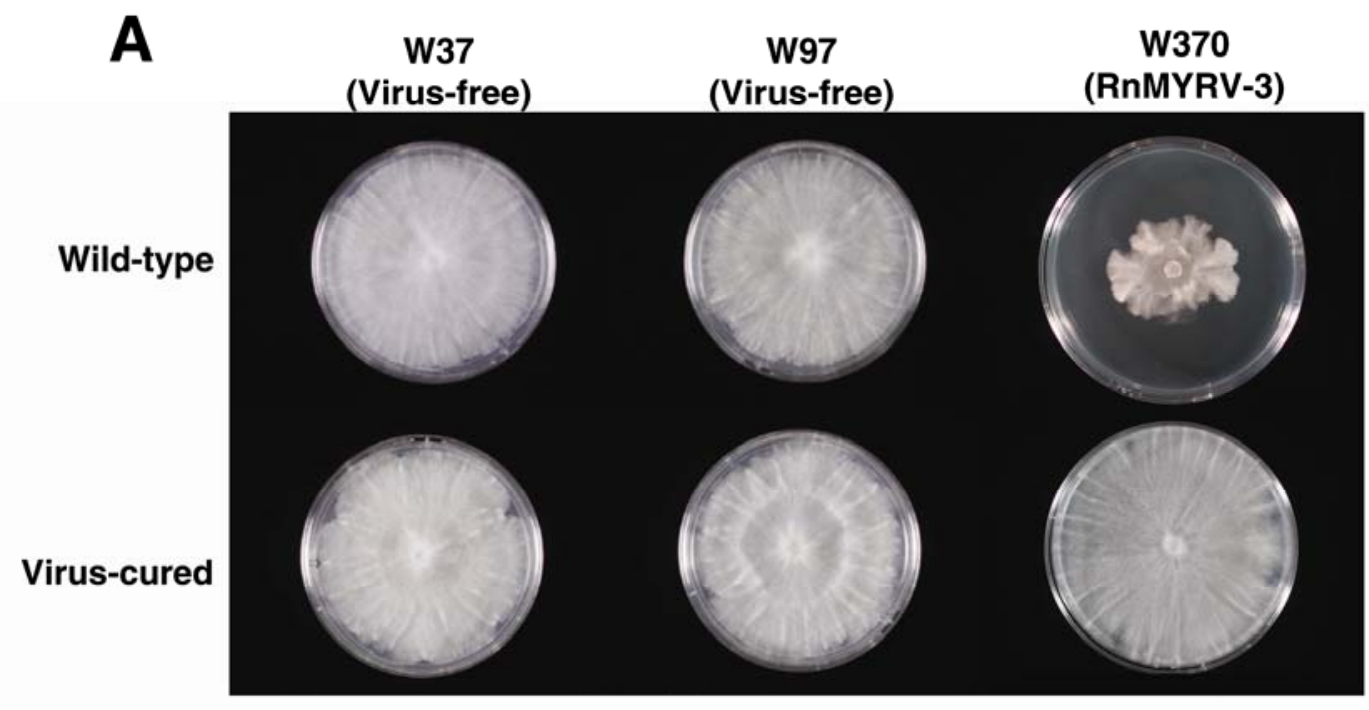

B

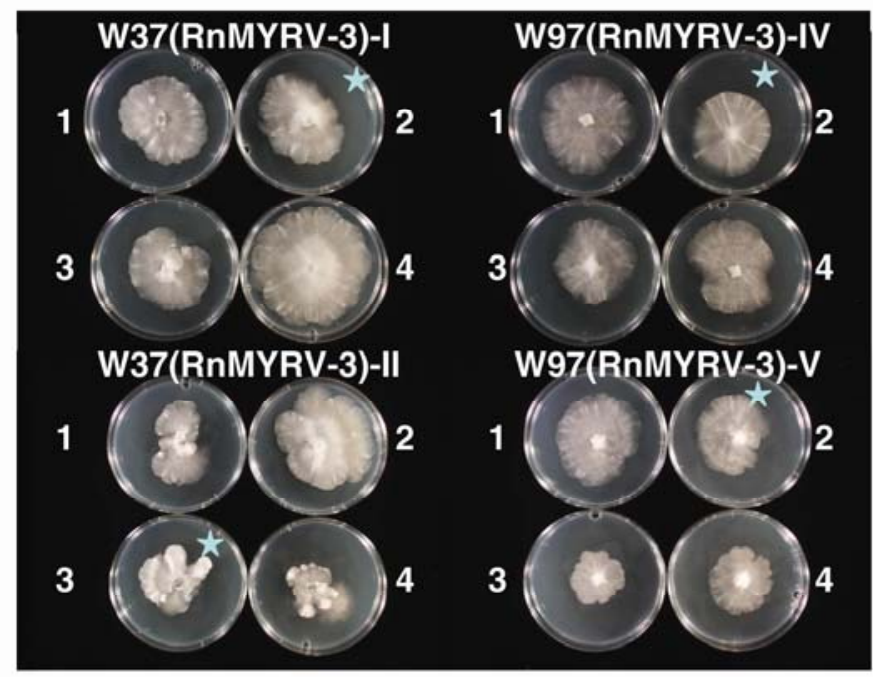

Primary cultures

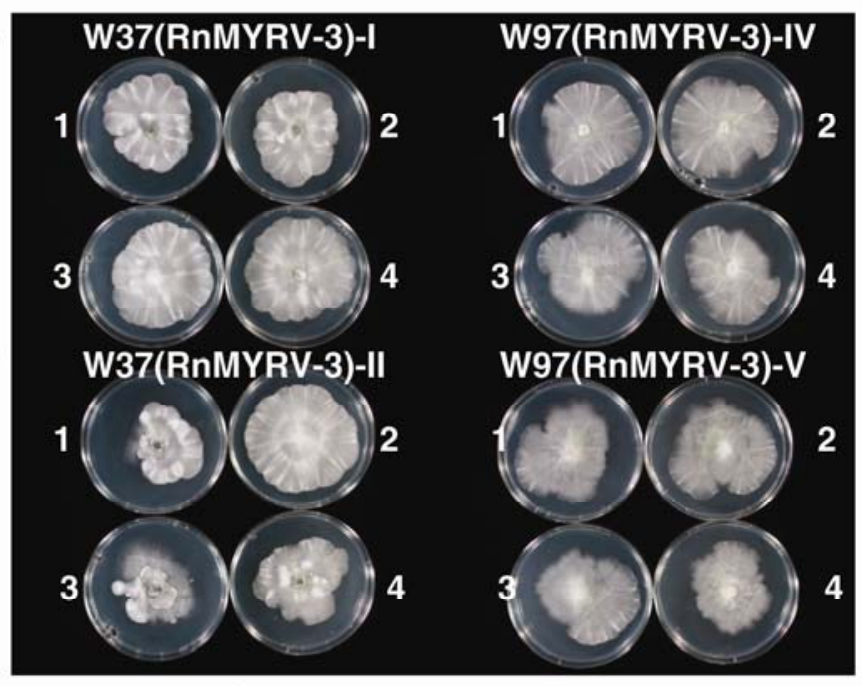

Secondary cultures

C

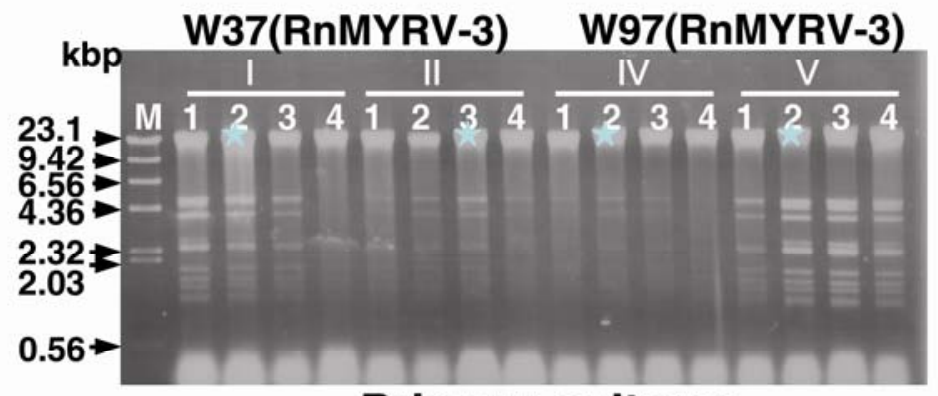

Primary cultures

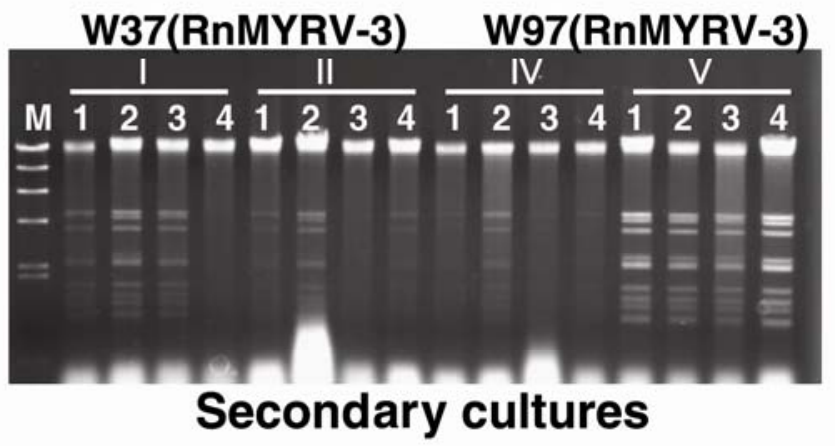

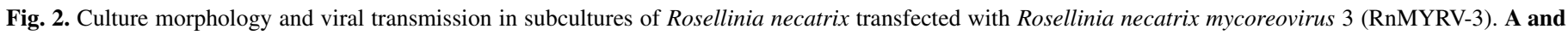

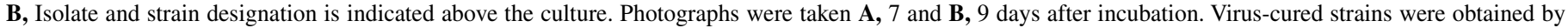

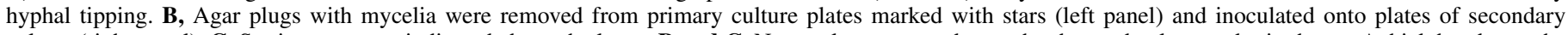

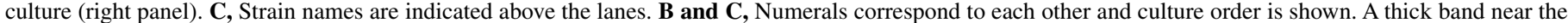

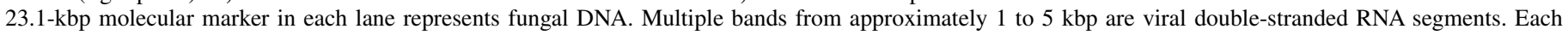
lane contains $1 / 50$ of extracts from $3.5 \mathrm{~cm}$ square culture on cellophane. 
Wild-type W37 and W97 exhibited approximately equivalent radial growth on PDA plates, while virus-transfected strains had dense mycelia and reduced growth, as did W370, but in addition to dispersed, fast-growing mycelia in single cultures. Morphological restoration in virus-cured strains obviously indicated that the change was incited by virus infection. The mixture of colony morphology represents one of the symptoms caused by RnMYRV-3, because the same morphology was observed in W370 when cultured from stocked mycelia at $4{ }^{\circ} \mathrm{C}$ (data not shown). Although $C$. parasitica infected with CpMYRV-1 showed constant and uniform morphological changes (11), symptoms of RnMYRV-3 were variable on transfected strains of $R$. necatrix and virus intensity sometimes did not coincide with colony morphology. We examined multiple sets of materials but the relationship was not evident.

The distribution of RnMYRV-3 in single colonies was not uniform in transfected strains of W37 and W97, and in isolate W370. Viral dsRNA was quantitatively compared based on the calibration with total DNA of $R$. necatrix, given that total DNA is expected to reflect the number of disrupted cells. As shown in Figure 4, the ratio of dsRNA to the amount of total DNA was different in single colonies of RnMYRV-3-infected strains, indicating the variable load of the virus in fungal cells. We also examined RnPV-1/W8-transfected strains of W37 and W97 to find that the virus was evenly distributed (data not shown). This uneven distribution is characteristic of the combination of RnMYRV-3 and $R$. necatrix. In addition, subcultures from the colonies de- veloped beneath cellophane on a $12 \mathrm{~cm}$ square plate (Figs. 3 and 4) showed viral distribution patterns different from those of mycelia above cellophane. The presence of RnMYRV-3 was variable in the subcultures even though virus-confirmed areas were used (data not shown). The virus is considered to distribute in a mosaic pattern in single colonies, i.e., virus-containing and virus-free portions. It is well known that plant viruses distribute unevenly in systemically infected plant hosts and even in single mosaic leaves: green and yellow islands containing less and more virus, respectively $(30,31)$. This unequal distribution of plant viruses may be explained by virus attack versus host defense via RNA silencing (14). It is interesting to analyze the mechanism underlying the uneven mycovirus distribution in single colonies.

RnMYRV-3 horizontal transmission was inefficient, but this observation contrasted with CpMYRV-1, in which whole recipient colonies exhibited symptoms of virus infection in dual culture plates (11). In the case of $R$. necatrix, the donor was sometimes surrounded by the recipient when they were inoculated at the same time, resulting in limited viral transmission near the contact line, as was the case with Kanematsu et al. (20). Viral spread in mycelia may simply depend on the rate of viral replication, because cell-to-cell movement proteins, found commonly in plant viruses, have never been reported from mycoviruses (5). Hyphal growth may be much faster than viral replication and mycelial spread of RnMYRV-3.

Inhibition of horizontal transmission by the fungal host is also possible. RnMYRV-3 transfected strains sometimes showed in-
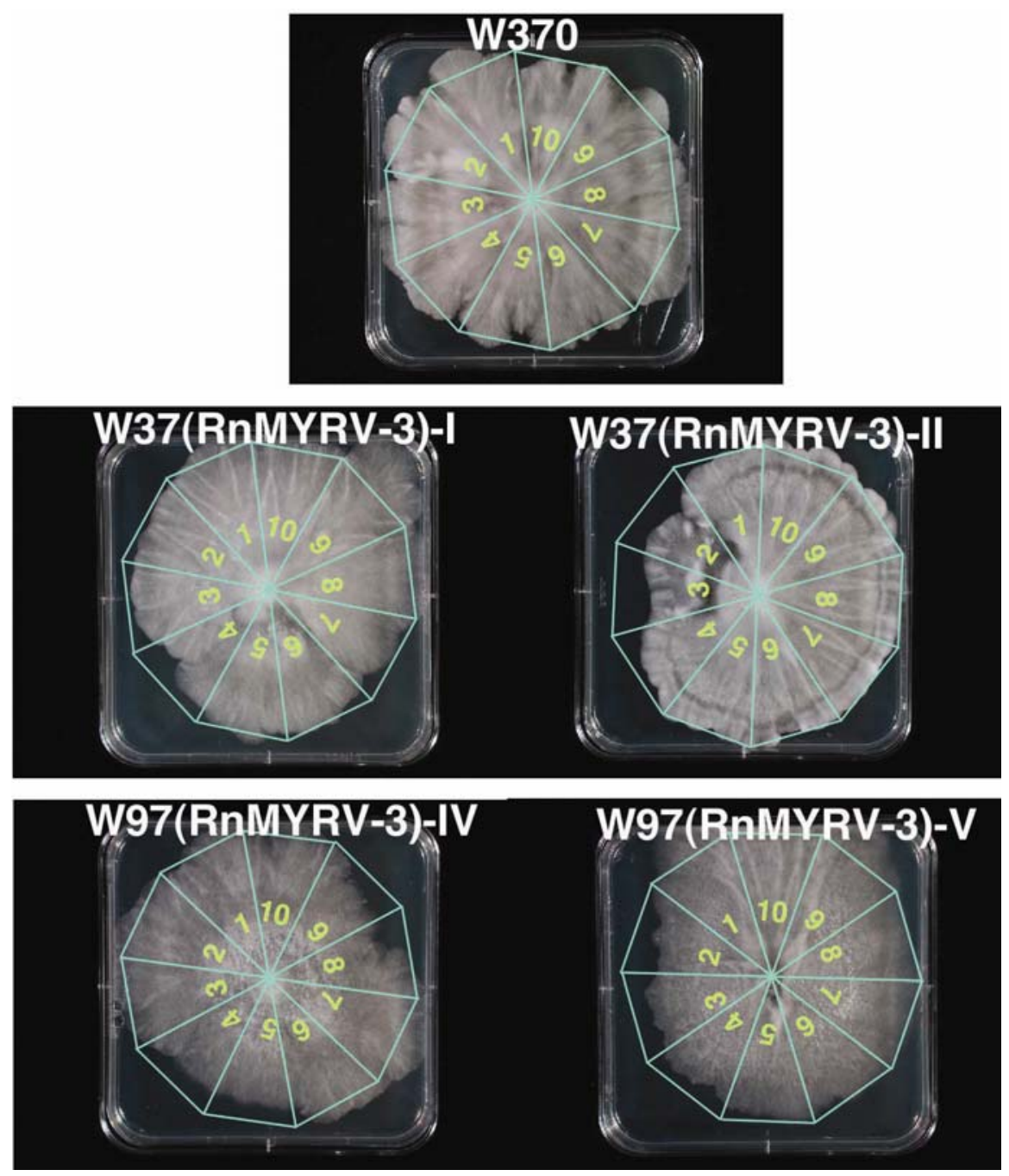

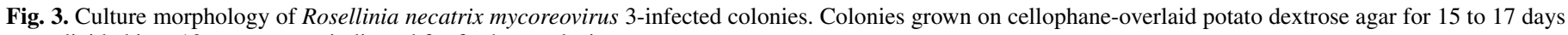
were divided into 10 segments as indicated for further analysis. 
compatibility-like reactions (broad pigmented line production between early contacted area or thin mycelia along the whole contact line) even when the combination was compatible (data not shown). No such reaction was observed with RnPV-1/W8, which was latent in $R$. necatrix. Cell death after contact with hypovirusinfected hyphae was reported in $C$. parasitica (3). A certain function may operate to inhibit the transmission of harmful agents through hyphal fusion in $R$. necatrix because this fungus mainly develops mycelia and never propagates from conidia in the orchard (24).

The virulence of $C$. parasitica strains was assayed using apple fruits $(8,11)$, and the same method was employed for the virulence of $R$. necatrix for the first time to our knowledge. The method certainly reflected the effect of virus on $R$. necatrix, because lesions caused by W370, naturally infected with RnMYRV3 , were smaller than those by their virus-cured counterparts. The level of virulence in $R$. necatrix isolates is usually estimated with yellow lupins (35) or apple seedlings (20), which takes 2 months to obtain results (20). In addition, the virus sometimes disappeared during the preparation of the inoculum and fungal establishment on plants. We consequently used mycelial agar plugs from cultures on cellophane-PDA as the inoculum so that the presence of dsRNA may easily be confirmed in the colony.

RnMYRV-3 was shown previously to be the causal factor of hypovirulence in the field isolate W370 infected with the virus (20). The smaller lesion size on apples by virus-transfected strains compared with wild-type isolates or virus-cured isolates derived from transfected strains (Fig. 6; Table 2) demonstrates that the virus contributes to the reduction of virulence for other $R$. necatrix isolates with different somatic compatibilities. Kanematsu et al. (20) described that the virulence correlated to mycelial growth on the host plants in soil. The mycelial growth rate in soil should also be reflected in apples. The reduction ratio in lesion sizes differed among strains derived from the same isolates. This may
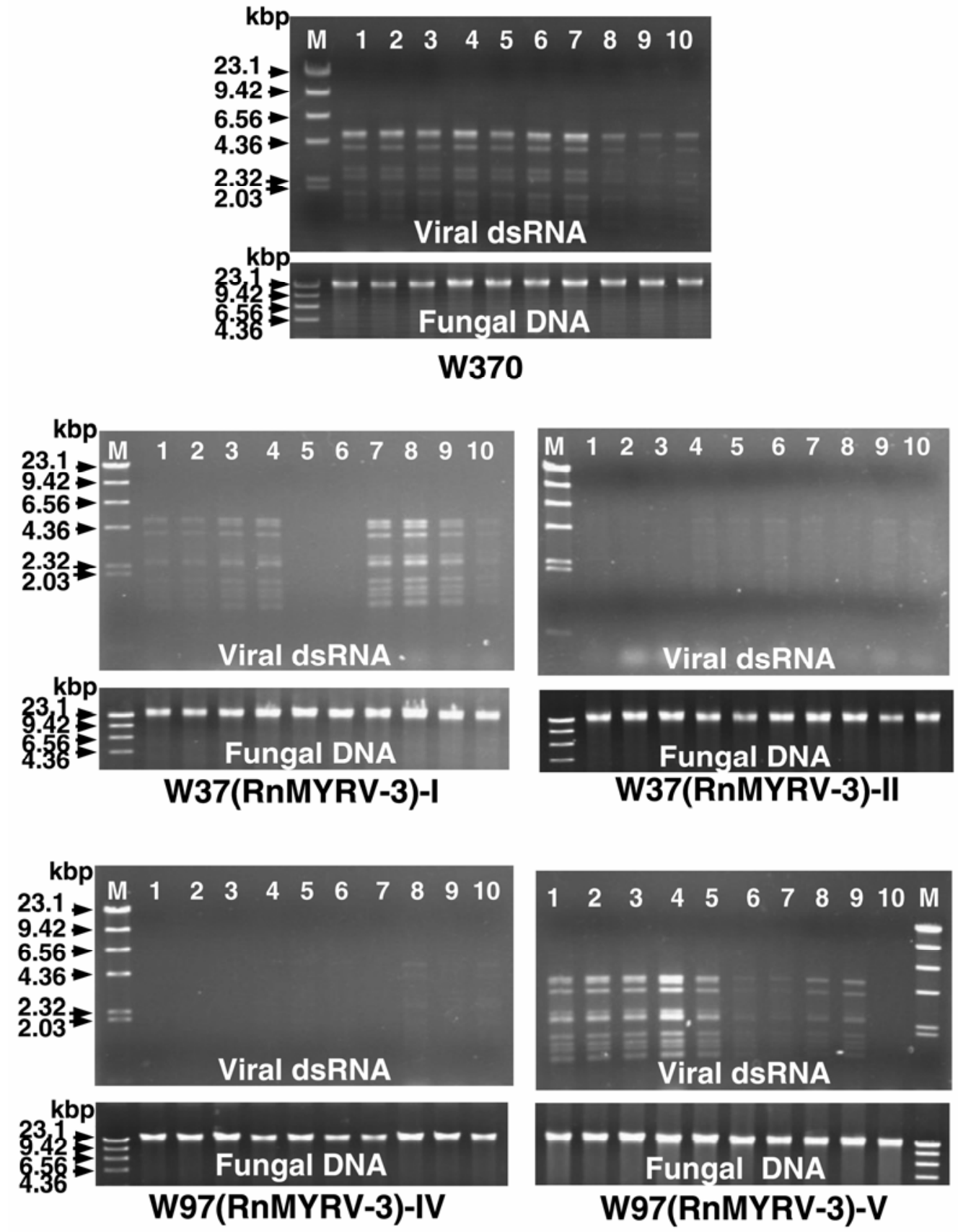

Fig. 4. Viral distribution in single colonies of Rosellinia necatrix. Two micrograms of total DNA was electrophoresed in each lane (lower panels), and doublestranded RNA fraction from those containing $4 \mu \mathrm{g}$ of total DNA fraction (upper panels). Numerals of lanes correspond to those on colony segments in Figure 3. 


\section{A w37hyg/ W37(RnMYRV-3)}

\section{W97hyg/ W97(RnMYRV-3)}

\section{RT37-1/W370}

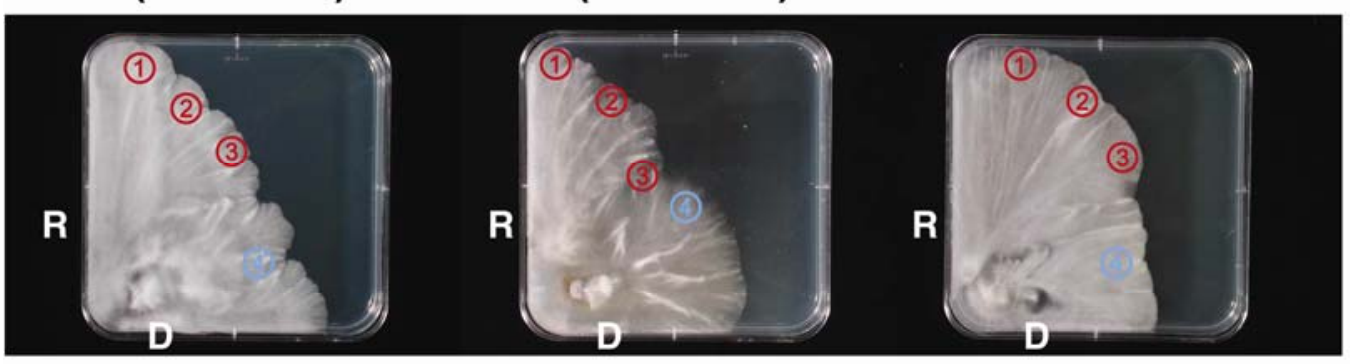

W37hyg/W370

W97hyg/W370

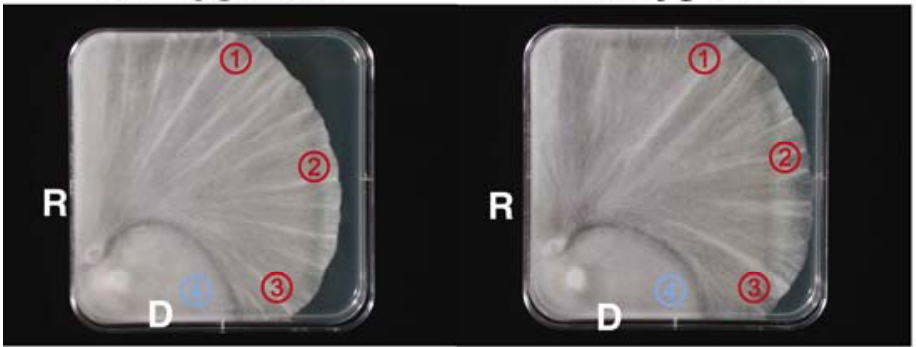

B W37hyg/ W37(RnMYRV-3)
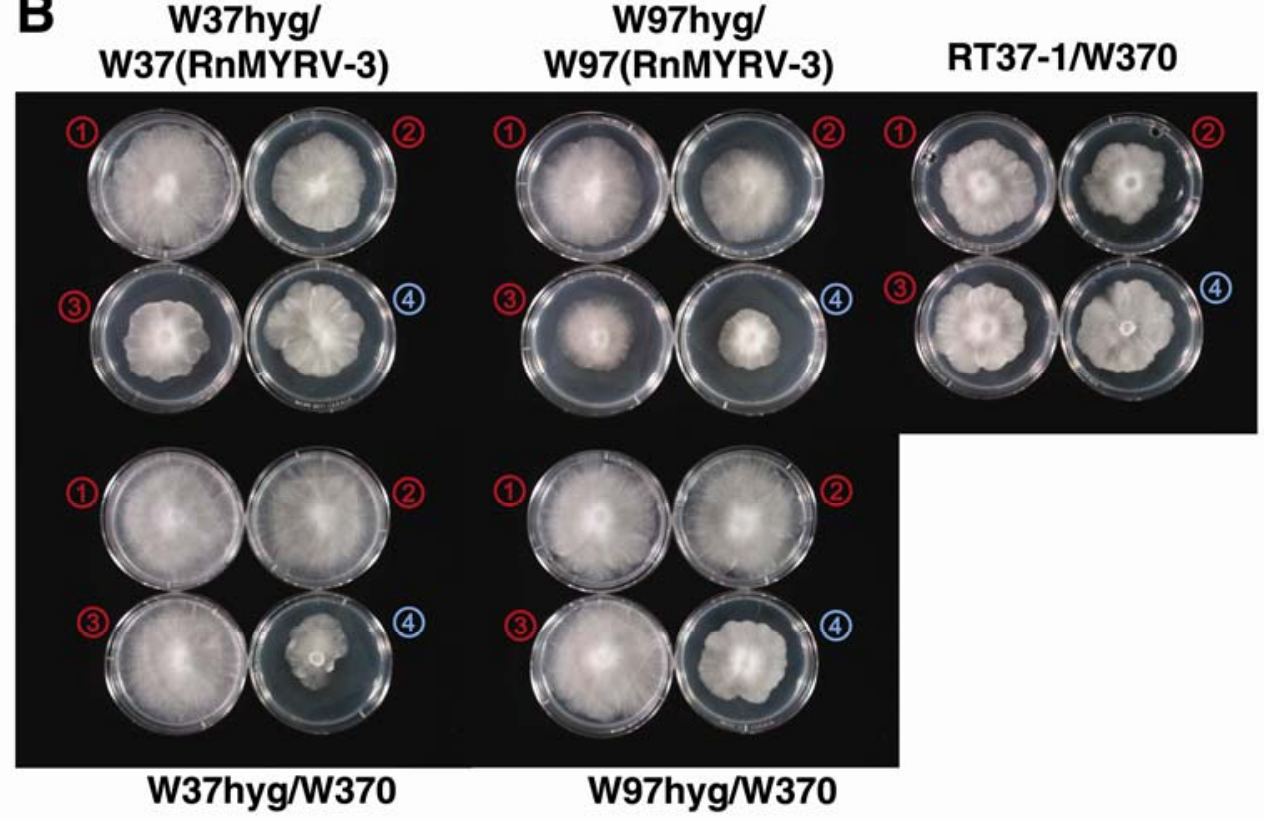

W37hyg/W370

W97hyg/W370

C

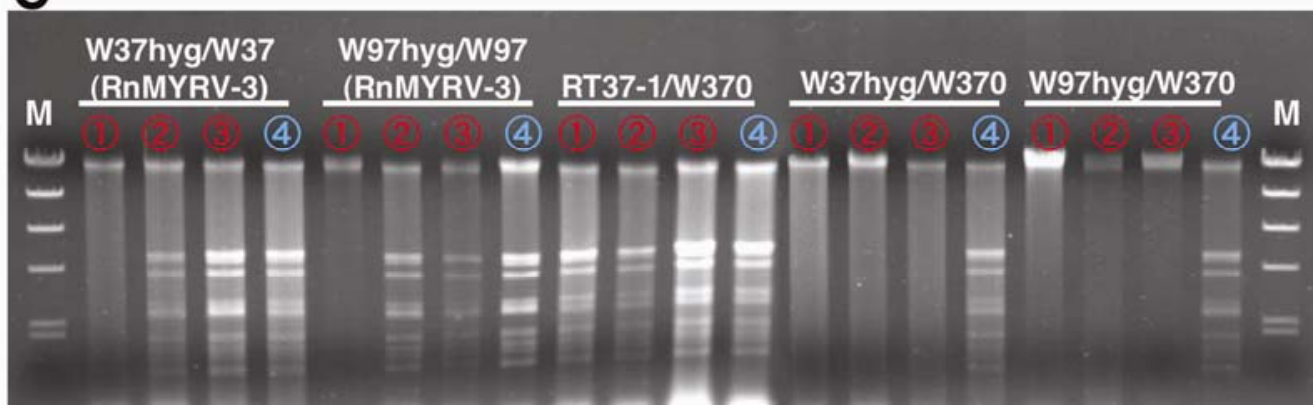

Fig. 5. Viral transmission in paired culture of Rosellinia necatrix. Virus transmission to virus-free recipients (W37hyg and W97hyg) from their respective counterparts ([W37(Rosellinia necatrix mycoreovirus 3 (RnMYRV-3)]) and W97[RnMYRV-3]) or from incompatible isolate W370. A, Paired isolate designation is indicated above each culture plate as recipient/donor: R, virus-free recipients transformed with the $h p h$ gene; and D, donors infected with RnMYRV-3. Mycelial plugs numbered 1 to 3 from the recipient colonies (indicated in red) and plugs numbered 4 from donor colonies (indicated in blue) were subcultured on potato dextrose agar (PDA) with and without hygromycin, respectively. B, Five-day-old PDA subcultures from plugs indicated in A. Strain designation is shown above and below the panel. C, Double-stranded RNA extracted from colonies in B. Pair designation is indicated above the lanes. Lane number indicates colony numeral as shown in $\mathbf{B}$. Each lane contains $1 / 50$ of extracts from $3.5 \mathrm{~cm}$ square culture on cellophane. 
reflect the variability in viral density within single colonies as observed in Figures 3 and 4. Although the results indicated that the reduction of virulence of $R$. necatrix by RnMYRV-3 was evident, it is difficult to use this virus as a biological control at this stage because of its instability in host fungus.

Mycoreoviruses reported from $R$. necatrix and $C$. parasitica were both infectious as purified virus particles. In our case, the

TABLE 2. Lesion size on apple fruits caused by virus-transfected strains and by their virus-free counterparts of Rosellinia necatrix

\begin{tabular}{lcc}
\hline & \multicolumn{2}{c}{ Lesion area $\left(\mathrm{cm}^{2}\right)^{\mathrm{a}}$} \\
\cline { 2 - 3 } Isolate and strain & Experiment 1 & Experiment 2 \\
\hline W370 & $3.30 \pm 0.35$ & $4.26 \pm 0.14$ \\
W370-free $^{\mathrm{b}}$ & $8.61 \pm 0.94$ & $9.06 \pm 0.38$ \\
W37 & $8.04 \pm 0.23$ & $8.63 \pm 0.35$ \\
W37(RnMYRV-3)-I & $2.34 \pm 1.15$ & $2.17 \pm 0.48$ \\
W37(RnMYRV-3)-II & $4.90 \pm 0.56$ & $4.13 \pm 0.57$ \\
W37(RnMYRV-3)-I-free & $9.60 \pm 0.13$ & $7.68 \pm 0.38$ \\
W97 & $8.66 \pm 0.59$ & $8.37 \pm 0.35$ \\
W97(RnMYRV-3)-IVc & $5.37 \pm 0.18$ & $6.67 \pm 0.33$ \\
W97(RnMYRV-3)-V $^{\mathrm{c}}$ & $3.05 \pm 0.30$ & $4.93 \pm 0.18$ \\
W97(RnMYRV-3)-V-free & $9.23 \pm 0.91$ & $8.97 \pm 0.47$ \\
\hline
\end{tabular}

${ }^{\text {a }}$ Lesion area $=1 / 2$ lengthwise $\times 1 / 2$ crosswise $\times 3.14$. Data are shown as means \pm standard deviation of three independent replicates.

b Virus-cured strain.

c Virus-transfected strain. Numerals indicate strain number. instability of the reovirus may explain the extent of compatibility with the host fungus. The disappearance of RnMYRV-3 is related to irregular viral distribution and difficulty in transmission. If virus-free sectors appear, they are likely to spread without viral transmission. In addition, inconsistency between viral distribution and fungal colony morphology masks viral decrease. Viral instability may be related to host defense responses toward virus invasion. Further analysis is needed to resolve the mechanisms for these interesting interactions.

\section{ACKNOWLEDGMENTS}

This research was supported by the program for Promotion of Basic Research Activities for Innovative Biosciences (BRAIN), and the program for JSPS Research Fellowships for Young Scientists. We thank N. Matsumoto for critical review of an early draft, and N. Suzuki for helpful comments on the manuscript. Contribution no. 1428 of the National Institute of Fruit Tree Science, NARO.

\section{LITERATURE CITED}

1. Anagnostakis, S. L. 1982. Biological control of chestnut blight. Science 215:466-471.

2. Arakawa, M., Nakamura, H., Uetake, Y., and Matsumoto, N. 2002. Presence and distribution of double-stranded RNA elements in the white root rot fungus Rosellinia necatrix. Mycoscience 43:21-26.

3. Biella, S., Smith, M. L., Aist, J. R., Cortesi, P., and Milgroom, M. G. 2002. Programmed cell death correlates with virus transmission in a filamentous fungus. Proc. R. Soc. Lond. B. Biol. Sci. 269:2269-2276.

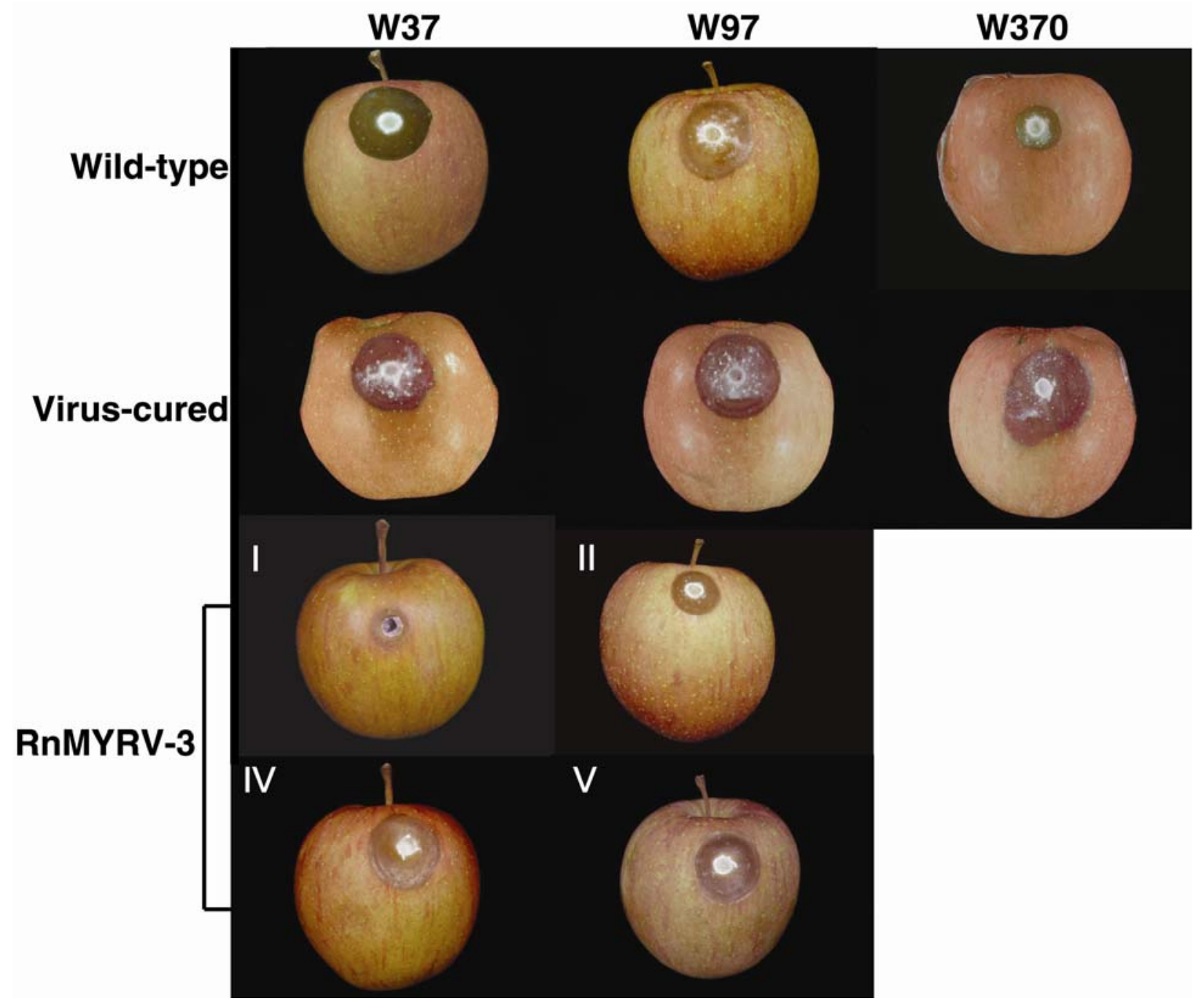

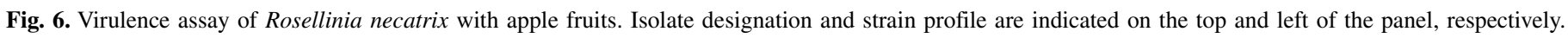
Virus-transfected strain number is indicated on the left corner of each picture. Photographs were taken 3 weeks after inoculation. 
4. Brasier, C. M. A. 1983. A cytoplasmic ally transmitted disease of Ceratocystis ulum. Nature 305:220.

5. Buck, K. W. 1998. Molecular variability of viruses of fungi. Pages 53-72 in: Molecular Variability of Fungal Pathogens. CAB International, Wallingford, Oxfordshire.

6. Chu, Y. M., Jeon, J. J., Yea, S. J., Kim, Y. H., Yun, S. H., Lee, Y. W., and Kim, K. H. 2002. Double-Stranded RNA Mycovirus from Fusarium graminearum. Appl. Environ. Microbiol. 68:2529-2534.

7. Enebak, S. A., MacDonald, W. L., and Hillman, B. I. 1994. A hypovirulent isolate of Cryphonectria parasitica with multiple genetically unique dsRNA elements. Mol. Plant-Microbe Interact. 7:590-595.

8. Fulbright, D. W. 1984. Effect of eliminating dsRNA in hypovirulent Endothia parasitica. Phytopathology 74:722-724.

9. Ghabrial, S. A., Buck, K. W., Hillman, B. I., and Milne, R. G. 2005. Family Partitiviridae. Pages 581-590 in: Virus Taxonomy, Eighth Report of the International Committee on Taxonomy of Viruses. Academic Press, San Diego, CA.

10. Ghabrial, S. A., Jiang, D., and Caston, J. R. 2005. Family Chrisoviridaee. Pages 591-595 in: Virus Taxonomy, Eighth Report of the International Committee on Taxonomy of Viruses. Academic Press, San Diego, CA.

11. Hillman, B. I., Supyani, S., Kondo, H., and Suzuki, N. 2004. A reovirus of the fungus Cryphonectria parasitica that is infectious as particles and related to the coltivirus genus of animal pathogens. J. Virol. 78:892-898.

12. Hillman, B. I., and Suzuki, N. 2004. Viruses of chestnut blight fungus, Cryphonectria parasitica. Adv. Virus Res. 63:423-472.

13. Huang, S., and Ghabrial, S. A. 1996. Organization and expression of the double-stranded RNA genome of Helminthosporium victoriae $190 \mathrm{~S}$ virus, a Totivirus infecting a plant pathogenic filamentous fungus. Proc. Natl. Acad. Sci. USA 93:12541-12546.

14. Hull, R. 2002. Pages 403-408 in: Matthews' Plant Virology. 4th ed. Academic Press, San Diego, CA.

15. Ikeda, K., Nakamura, H., Arakawa, M., and Matsumoto, N. 2004. Diversity and vertical transmission of double-stranded RNA elements in root rot pathogens of trees, Helicobasidium mompa and Rosellinia necatrix. Mycol. Res. 108:626-634.

16. Ikeda, K., Nakamura, H., and Matsumoto, N. 2003. Hypovirulent strain of the violet root rot fungus Helicobasidium mompa. J. Gen. Plant Pathol. 69:385-390.

17. Ikeda, K., Nakamura, H., and Matsumoto, N. 2005. Comparison between Rosellinia necatrix isolates from soil and diseased roots in terms of hypovirulence. FEMS Microbiol. Ecol. 54:307-315.

18. Jian, J., Lakshman, D. K., and Tavantzis, S. M. 1997. Association of distinct double-stranded RNAs with enhanced or diminished virulence in Rhizoctonia solani infected potato. Mol. Plant-Microbe Interact. 10:1002-1009.

19. Kanadani, G., Date, H., and Nasu, H. 1998. Effect of Fluazinam soildrench on white root rot of grapevine. Ann. Phytopathol. Soc. Jpn. 64:139-141.

20. Kanematsu, S., Arakawa, M., Oikawa, Y., Onoue, M., Osaki, H., Nakamura, H., Ikeda, K., Kuga-Uetake, Y., Nitta, H., Sasaki, A., Suzaki, K., Yoshida, K., and Matsumoto, N. 2004. A reovirus causes hypovirulence of Rosellinia necatrix. Phytopathology 94:561-568.

21. Lakshman, D. K., Jian, J., and Tavantzis, S. M. 1998. A double-stranded RNA element from a hypovirulent strain of Rhizoctonia solani occurs in DNA form and is genetically related to the pentafunctional AROM protein of the shikimate pathway. Proc. Natl. Acad. Sci. USA 95:64256429.

22. Matsumoto, N. 1998. Biological control of root disease with dsRNA based on population structure of pathogens. Jpn. Agric. Res. Quart. 32:31-35.

23. Mertens, P. P. C., Duncan, R., Attoui, H., and Dermody, T. S. 2005. Family Reoviridae. Pages 447-454 in: Virus Taxonomy, Eighth Report of the International Committee on Taxonomy of Viruses. Academic Press, San Diego, CA.

24. Nakamura, H., Uetake, Y., Arakawa, M., Okabe, I., and Matsumoto, M. 2000. Observations the teleomorph of the white root rot fungus, Rosellinia necatrix, and related fungus, Rosellinia aquia. Mycoscience 41:503-507.

25. Nuss, D. L., Hillman, B. I., Rigling, D., and Suzuki, N. 2005. Family Hypoviridae. Pages 597-601 in: Virus Taxonomy, Eighth Report of the International Committee on Taxonomy of Viruses. Academic Press, San Diego, CA.

26. Osaki, H., Wei, C. Z., Arakawa, M., Iwanami, T., Nomura, K., Matsumoto, N., and Ohtsu, Y. 2002. Nucleotide sequences of doublestranded RNA segments from a hypovirulent strain of the white root rot fungus Rosellinia necatrix: Possibility of the first member of the Reoviridae from fungus. Virus Genes 25:101-107.

27. Preisig, O., Moleleki, N., Smit, W. A., Wingfield, B. D., and Wingfield, M. J. 2000. A novel RNA mycovirus in a hypovirulent isolate of the plant pathogen Diaporthe ambigua. J. Gen. Virol. 81:3107-3114

28. Sasaki, A., Kanematsu, S., Onoue, M., Oyama, Y., and Yoshida, K. 2006. Infection of Rosellinia necatrix with purified viral particles of a member of Partitiviridae (RnPV1-W8). Arch. Virol. 151:697-707.

29. Sasaki, A., Miyanishi, M., Ozaki, K., Onoue, M., and Yoshida, K. 2005. Molecular characterization of a partitivirus from the plant pathogenic ascomycete Rosellinia necatrix. Arch. Virol. 150:10691083.

30. Suzuki, N., Kudo, T., Shirako, Y., Ehara, Y., and Tachibana, T. 1989 Distribution of cylindrical inclusion, amorphous inclusion and capsid protein of watermelon mosaic virus 2 in systemically infected pumpkin leaves. J. Gen. Virol. 70:1085-1091.

31. Suzuki, N., Sugawara, M., Kusano, T., Mori, H., and Matsuura, Y. 1994. Immunodetection of rice dwarf phytoreoviral proteins in both insect and plant hosts. Virology 202:41-48.

32. Suzuki, N., Supyani, S., Maruyama, K., and Hillman, B. I. 2004 Complete genome sequence of Mycoreovirus-1/Cp9B21, a member of a novel genus within the family Reoviridae, isolated from the chestnut blight fungus Cryphonectria parasitica. J. Gen. Virol. 85:34373448.

33. Sztejnberg, A. 1991. Rosellinia (Dematophora) root rot. Pages 46-47 in: Compendium of Apple and Pear Disease. The American Phytopathological Society, St. Paul, MN.

34. Terai, Y. 1995. Rosellinia (Dematophora) root rot. Pages 42-43 in: Compendium of Stone Fruit Disease. The American Phytopathological Society, St. Paul, MN.

35. Uetake, Y., Nakamura, H., Arakawa, M., Okabe, I., and Matsumoto, N. 2001. Inoculation of Lupinus luteus with white root rot fungus, Rosellinia necatrix, to estimate virulence. J. Gen. Plant Pathol. 67:285287.

36. Wei, C. Z., Osaki, H., Iwanami, T., Matsumoto, N., and Ohtsu, Y. 2003. Molecular characterization of dsRNA segments 2 and 5 and electron microscopy of a novel reovirus from a hypovirulent isolate, W370, of the plant pathogen Rosellinia necatrix. J. Gen. Virol. $84: 2431-2437$

37. Wei, C. Z., Osaki, H., Iwanami, T., Matsumoto, N., and Ohtsu, Y. 2004. Complete nucleotide sequences of genome segments-1 and 3 of Rosellinia anti-rot virus in the family Reoviridae. Arch. Virol. 149:773-777.

38. Wickner, R. B., Wang, C. C., and Patterson, J. L. 2005. Family Totiviridae. Pages 571-580 in: Virus Taxonomy, Eighth Report of the International Committee on Taxonomy of Viruses. Academic Press, San Diego, CA. 